\title{
Structural relations between programs and problems
}

\section{Report}

Author(s):

Engeler, Erwin

Publication date:

1975

Permanent link:

https://doi.org/10.3929/ethz-a-000072470

Rights / license:

In Copyright - Non-Commercial Use Permitted

Originally published in:

Berichte des Instituts für Informatik 15 
Eidgenössische Technische Hochschule

\section{Zürich}

\section{Institut für Informatik}

Erwin Engeler

Structural Relations between Programs and Problems 
STRUCTURAL RELATIONS BETWEEN PROGRAMS AND PROBLEMS

by Erwin Engeler

\section{Abstract}

By a problem in a universal first-order theory $\Gamma$ we understand a formula $\varphi(y)$ with a free variable $y$. The problem $\varphi(y)$ is solvable relative to auxillary problems $\psi_{1}(v), \ldots, \psi_{s}(v)$ if solution algorithms for the $\psi_{i}$ can be so composed as to yield all solutions of $\varphi$ in all models of $\Gamma$. The complexity of the composite algorithm is the number of times that the auxillary algorithms have to be called. A lower bound for the complexity of $\varphi$ is obtained by developing a generalized Galois theory for theories $\Gamma$ and problems $e p$ satisfying some reasonable restrictions; our lower bound is the logarithm of the order of the "Galois group" of $\varphi$.

Limited distribution notice:

This report is to be published in the Proceedings of the 5 th International Congress of Logic, Methodology and Philosophy of science as an invited address. As a courtesy to the intended publisher this report is distributed on request only. 
We begin by placing our investigationsinto a framework describing various activities by logicians in the field of the theory of computation.

As the basis is the mathematical structure on which computations are performed. This may be a relational structure such as the natural number system $\langle N, S, 0, \Rightarrow$ (for elementary recursion theory), a geometry (for various theories of geometrical constructions), etc. Mathematical structures also arise typically in computing as recursively defined data structures such as trees, lists, records and the like (where, in contrast to the relational structures mentioned, the body of mathematical knowledge about them is rather meager). We deal here with two aspects of the mathematical structure in question, namely the descriptive aspect, and the computational aspect.

The descriptive aspect of relational structures is dealt with by formalisms of classical (or intuitionistic) logic for finite or infinitary languages, for example; variants of $\lambda$-calculi have been found to be appropriate for recursively defined data structures. of course, the chaice of a descriptive formalism depends on the uses we forsee for it.

The computational aspect is customarily dealt with by various algorithmic (or 'programming') languages, of which the simplest define the classes of flowchart programs operating in relational structures, see section 3 below. Such languages can be enriched by allowing recursive procedures, counters and other features; the investigation of their relative strength is an interesting and important area of research. There is also the need to explain computations on objects of higher. type, on infinitary objects and on incompletely determined abjects, to deal with notions of nondeterminism and (asynchronous) paraliel computation. 
The goal of algorithmic logic is to establish and investigate relations between the formal descriptive and computational aspects of mathematical structures. It is apparent that such relations wait to be established on various levels. One particular example is between flowchart programs and constructive fragments of $L_{\omega}{ }_{\omega} w$ which we originated and which Rasiowa's group developed into a unified and well understood tool (see e.g. Salwicki's paper for this Congress).

A central concern of algorithmic logic is the investigation of the solvability of alqorithmic problems. The classical paradigms of an algorithmic problem are geometrical construction problems: Given a figure $F$ (consisting of finitely many points and lines) construct an algorithm $\pi$ which by repeated use of ruler and compass, produces a figure $F^{\prime}$ satisfying some prescribed condition $\varphi\left(F^{\prime}\right)$. For example, to construct the perpendicular bisector $I$ of two points $P, Q$ we seek an algorithm $\pi(P, Q)$ such that if $l:=\pi(P, Q)$ then

$$
1 \perp(P, Q) \wedge d(P, I)=d(Q, I) \text {. }
$$

It is apparent, that to make the concept of an algorithmic problem precise, we shall have to define precisely what we admit as an "algorithm" and what language we choose our "condition $\varphi$ "from. As it is possible to develop an interesting theory already with the simplest such choices, we shall concentrate here an computing in relational structures by means of flowchart programs and formulating problems by quantifier-free first-order formulas.

Dur main goal here is to discuss the solvability of algorithmic problems relative to some auxillary capabilities. In geometry we may, for example, envision an algebraic or transcendental curve as given and use it - by intersecting it with canstructed lines to solve problems not solvable without (e.g. trisection of angles, squaring the circle). In algebra, we may admit as additional capabilities the extraction of $k$-th roots. For the classical case, 
Galois theory is the appropriate tool; our method here is to use a generalization of Galois theory to deal with the more generalized situation as far as possible.

In computation theory we are not anly interested in (relative) solvability in principle, but also in the feasibility of alqorithms. Concretely, we should like to have good upper and lower bounds for the number of aperations to be performed for the solution of a given problem. As a rule, upper bounds require intelligent programming, lower bounds generally call for rather non-trivial mathematics. The main result of the present paper is a general theorem giving lower bounds for relative solution algorithms.

2. The group of a problem

We recall briefly some of the definitions and results of [1]. Let $L$ be a first-order language for relational structures of a fixed finite similarity type. $L_{0}$ is the set of basic (i.e. negated or unnegated atomic) formulas of $L$. If $a_{1}, \ldots, a_{m}$ are new individual constants we let $L\left(a_{1}, \ldots, a_{m}\right), L_{0}\left(a_{1}, \ldots, a_{m}\right)$ denote the carresponding augmented languages. Lower case greek letters $\varphi, \psi, \rho, \ldots$ denote formulas; sets of formulas are denoted by capital greek letters. We may indicate the occurrence of additional individual constants in the style $\varphi\left(a_{1}, \ldots, a_{m}\right)$, $\Delta\left(a_{1}, \ldots, a_{m}\right)$. The provability relation is indicated by $\Gamma \vdash \varphi$, the satisfaction relation by $\mathfrak{A} \equiv c p$, where $\mathbb{U}$ is a relational structure of the given type and $\phi$ a closed formula of $L$. By a diagram in $L$, respectively $L\left(a_{1}, \ldots, a_{m}\right)$, we mean a consistent subset of $L_{0}$, respectively $L_{0}\left(a_{1}, \ldots, a_{m}\right)$ which contains, for each atomic formula $\alpha$, either $\alpha$ or $\neg \alpha$. A theory $\Gamma$ in $L$ is a consistent set of closed formulas of $L$; it is universal, if $\Gamma$ consists of universally quantified boolean combinations of atomic formula exclusively. 
A theory $\Gamma$ is said to have the amalqamation property if for any models $\mathfrak{U}, \mathfrak{B}_{1}$ and $\mathfrak{B}_{2}$ and any injections of $\mathbb{U}$ into $\mathfrak{B}_{1}$ and $B_{2}$ there is a model $\mathfrak{S}$ of $\Gamma$ and injections of $B_{1}$ and $B_{2}$ into $\mathbb{s}$ such that the diagram

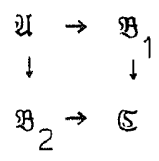

commutes. The importance of the amalgamation property for Galois theory has been observed by Jónsson [2], the general concept is due to Fraissé. The class of theories with the amalgamation property is wide enough to attract a general theory: it includes fields, differential fields, geometries, boolean algebras, lattices, etc.. A particularly useful class consists of those theories whose models have the same fixed finite cardinality, where the property is trivially satisfied.

Let $\Gamma$ be a universal theory. By a problem, we understand a quantifier-free formula $\varphi\left(y_{1}, \ldots, y_{k}\right)$ in some extension $L\left(a_{1}, \ldots, a_{m}\right)$ of $L$, with exactly the free variables $y_{1}, \ldots, y_{k}$ indicated. For notational convenience we choose $m=k=1$; the reader can easily supply the modifications in definitions and proofs required below. The spectrum of $c(y)$ in $\Gamma$ is the set of all $n \in \mathbb{N} v\{\infty\}$ for which there exists a diagram $\Delta(a)$, consistent with $\Gamma$, such that in all models of $\Gamma u \Delta(a)$ the problem $\varphi(y)$ has exactly $n$ solutions; $n$ is called the deqree of $\Delta(a)$ in this case. If $\infty \notin$ spectrum $(\varphi)$ then the spectrum is finite (by completeness theorem), its maximal element is then called the deqree of $\varphi$. We shall be concerned here exclusively with problems of finite degree. The theory, however, generalizes.

Let $\Delta(a)$ be a diagram in $\Gamma, \varphi(y)$ a problem and $n$ the degree of $\Delta(a)$ for $\varphi(y)$. Consider new individual constants $b_{1}, \ldots, b_{n}$ and a diagram $\Delta\left(a, b_{1}, \ldots, b_{n}\right)$ which extends $\Delta(a)$, and is consistent with $\Gamma$. The extended diagram is called a splitting diagram (for $\varphi$ over $\Delta(a)$ ) if 
(i) $\quad \Gamma \cup \Delta\left(a, b_{1}, \ldots, b_{n}\right)+\omega\left(b_{i}\right), i=1, \ldots, n$;

(ii) $b_{i} \neq b_{j} \in \Delta\left(a, b_{1}, \ldots, b_{n}\right), i \neq j$.

The group of the problem $o(y)$ is defined by

$G_{\Delta(a)}(\varphi)=\left\{s \in s_{n}: \Gamma \cup \Delta\left(a, b_{1}, \ldots, b_{n}\right) \vdash \rho \equiv \rho^{s}\right.$ for all $\left.\rho \in L_{0}\left(a, b_{1}, \ldots, b_{n}\right)\right\}$

where $S_{n}$ is the set of all permutations of $\{1,2, \ldots, n\}$ and $\rho^{s}$ results from $\rho$ by substituting $b_{s}(i)$ for all occurrencies of $b_{i}$ in $\rho, i=1,2, \ldots, n$. If $\Gamma$ has the amalgamation property then one can show (see [1] for details), that $G_{\Delta(a)}(\varphi)$ is indeed a group and does not depend on the particular choice of a splitting diagram.

The group of a problem can also be characterized, as in classical Galois theory, by a suitable group of autamorphisms. Namely, let $\mathfrak{U}(a), \mathcal{U}\left(a, b_{1}, \ldots, b_{n}\right)$ be, respectively, the minimal models of $\Gamma \cup \Delta(a)$ and $\Gamma \cup \Delta\left(a, b_{1}, \ldots, b_{n}\right)$. (Such models exist by the universality of $\Gamma$.) Let $G\left(\mathscr{I}\left(a, b_{1}, \ldots, b_{n}\right) / \mathscr{I}(a)\right)$ be the group of automorphisms of $\mathfrak{A}\left(a, b_{1}, \ldots, b_{n}\right)$ which leave $\mathfrak{M}(a)$ pointwise fixed; then (see [1], corollary 6) we have

$$
G_{\Delta(a)}(\varphi) \cong G\left(\mathscr{U}\left(a, b_{1}, \ldots, b_{n}\right) / \mathscr{U}(a)\right) \text {. }
$$

We shall use both aspects of the group of $\varphi(y)$ interchangeably as the case may be.

For applications, it is sometimes convenient, indeed essential, to enlarge the group of $\omega(y)$ by relaxing the symmetry requirements. Namely, let $L^{*}$ be a subrange of $L$ which still contains the vocabulary of $\rho(y)$. Then $G_{\Delta(a)}^{*}(\varphi)=\left\{s \in S_{n}: \Gamma \cup \Delta\left(a, b_{1}, \ldots, b_{n}\right) \vdash \rho \equiv \rho^{s}\right.$ for all of $\left.L_{a}^{*}\left(a, b_{1}, \ldots, b_{n}\right)\right\}$ is obviously a (normal) extension of $G_{\Delta(a)}(\varphi)$. It is easy to define the appropriate automorphism group isomorphic to $G_{\Delta(a)}^{*}(\varphi)$.

Questions on how to actually determine the group of a problem will be treated in section 4 . 


\section{Relative solvability}

Let $\Gamma$ be a universal theory with the amalgamation property, fixed for the following. Let $L$ be the language of $\Gamma$, containing function symbols $f^{j}$, predicate symbols $R_{j}$ and individual constants $c_{j}$ altogether finitely many. Some, or all, of these symbols will now be associated to basic capabilities for performing constructions in models $\mathfrak{u}$ of $\Gamma$. We express these capabilities, using variables $x_{1}, x_{2}, \ldots$, in the form of basic instructions $x_{i}:=c_{j} \quad$;

$$
\begin{aligned}
& x_{i}:=f j\left(x_{k_{1}}, \ldots, x_{k_{n_{j}}}\right) ; \\
& \text { if } R_{j}\left(x_{k_{1}}, \ldots, x_{k_{m_{j}}}\right) \text { then } \ldots \text { else } \ldots \text {. }
\end{aligned}
$$

Such instructions are composed into some kind of programs, e.g. flowchart-proqrams whose unary nodes are labelled by instructions of the first or second kind and whose binary nodes are labelled by instructions of the third kind. By a path through a program we understand a (finite or infinite) sequence of symbols from the set

$$
\left\{i^{c_{j}}, i^{f_{k}^{j}}, \ldots, k_{n_{j}}, j R_{k_{1}}^{+}, \ldots, k_{m_{j}}, j^{R_{k}}, \ldots, k_{m_{j}}\right\} i, j, k_{1}, \ldots
$$

which describes a legal execution sequence of the program; $i^{c_{j}}$ denotes execution of $x_{i}:=c_{j}$, etc.. The central requirement of the programming language is the following: For every program and every diagram $\Delta$, consistent with $\Gamma$, the set

\{w: $w$ is a finite initial segment of a path through the program taken in some model of $\Gamma v \Delta\}$

is recursive relative to $\Delta$. Flowchart programs clearly have this property, so do recursive programs.

We employ programs to construct solutions for problems as follows: Let $c(y)$ be a problem of degree $n$ in $L$. Let $\pi$ be a program with output variables $y_{1}, \ldots, y_{n}$ whose instructions are chosen from the set of basic instructions. The program $\pi$ is said to solve the problem $\varphi(y)$ if for every diagram $\Delta$ consistent with $\Gamma$ 
and every model $\mathscr{U}$ of $\Gamma_{v} \Delta$ there is a finite legal path through the program $\pi$, such that the execution of $\pi$ along this path terminates with values $b_{1}, \ldots, b_{n}$ for $y_{1}, \ldots, y_{n}$ such that II $=\varphi\left(b_{i}\right), i=1, \ldots, n$, and the number of different $b_{i}$ is equal to the degree of $\Delta$. Clearly, these solutions will be the values of constant terms of $L$, and hence the group of $\varphi(y)$ is trivial in this case.

The situation becomes more interesting, if we add to the basic capabilities the (hypothetical) solvability of some auxillary problems with nontrivial groups and consider problems $\varphi(y)$ solvable using these added capabilities. - Concretely, let $\psi(a, v)$ be a problem of degree $m$ in $L(a)$, where $a$ is an individual constant which we shall use as a parameter. We introduce m unary function symbols and add the capabilities

$$
\left\{x_{i}:=g_{j}\left(x_{k}\right): j=1, \ldots, m\right\}_{i, k}
$$

to our programming language. For every diagram $\Delta(a)$ consistent with $\Gamma$ and every splitting diagram $\Delta\left(a, b_{1}, \ldots, b_{m}\right)$ for $\psi(a, v)$ over $\Delta(a)$ and model $\mathfrak{U}\left(a, b_{1}, \ldots, b_{n}\right)$ of $\Gamma \cup \Delta\left(a, b_{1}, \ldots b_{n}\right)$ the terms $g_{1}(a), \ldots, g_{m}(a)$ give an enumeration (perhaps with repetition: of the set $\left\{b_{1}, \ldots, b_{n}\right\}$. We say that $c(y)$ is solvable relative to $\psi(u, v)$ if there exists a program in the extended capabilities which solves $\varphi(y)$.

\{To have a concrete example, the reader is invited to think of $\Gamma$ as the theory of fields of characteristic zero, $\varphi(y)$ a polynomial equation $p(y)=0$ with rational coefficients and $\psi(u, v)$ the equation $v^{m}=u$. Relative solvability here would mean solvability of $p(y)=0$ by $m$-th radicals. $\}$ 
Let $\pi$ be a solution program for capabilities, and let $w$ be a finite path through $\boldsymbol{m}$ which is consistent with $\Gamma$, i.e. such that there is a model of $\Gamma$ in which execution of $\pi$ actually progresses along $w$. Let $k$ be the number of times a problem $\psi$ has to be solved along this path. We construct a sequence of diagrams

$\Delta \subseteq \Delta\left(a_{11}, \ldots, a_{1 m_{1}}\right) \subseteq \Delta\left(a_{11}, \ldots, a_{1 m_{1}}, a_{21}, \ldots, a_{2 m_{2}}\right) \subseteq \ldots \subseteq \Delta\left(a_{11}, \ldots, a_{k m_{k}}\right)$

corresponding to this path as follows: The first time the prablem $\psi$ needs to be solved along this path is after some instructians of the original programming language are executed. The problem, therefore, is posed in the form $\psi\left(\tau_{1}, v\right)$, where $T_{1}$ is a constant term of $L$. Let $\Delta\left(a_{1}, \ldots, a_{1_{m_{1}}}\right)$ be a splitting diagram of $\psi(T, v)$ over $\Delta$. The later diagrams are found the same way; $\Delta\left(a_{11}, \ldots, a_{p m_{p}}, a_{p+1}, \ldots, a_{p+1} m_{p+1}\right)$ is a splitting diagram of $\psi\left(\tau_{p+1}, v\right)$ aver $\Delta\left(a_{11}, \ldots, a_{p m_{p}}\right)$, where $\tau_{p+1}$ is a canstant term in $L\left(a_{11}, \ldots, a_{p m_{p}}\right)$. The numbers $m_{p}$ are the respective degrees of the problems $\psi\left(\tau_{p}, v\right), p=1, \ldots, k$. Finally, let $y_{1}, \ldots, y_{n}$ be the output variables of $\pi$. Since $\pi$ solves $p(y)$ along $w$ the values of $y_{1}, \ldots, y_{n}$ are determined and can be expressed as constant terms $\sigma_{1}, \ldots, \sigma_{n}$ of $L\left(a_{11}, \ldots, a_{k m k}\right)$.

For the thus defined sequence of diagrams we now determine a corresponding sequence of permutation groups. Let $P$ be the set of all permutations $t$ of $\left\{a_{11}, \ldots, a_{k m_{k}}\right\}$ such that $t\left(a_{i j}\right)=a_{i p}$ for some $p$. Then, for each $q=0,1, \ldots, k$, Iet

$$
\begin{aligned}
G_{q}=\{t \in P: & \Delta^{t}\left(a_{11}, \ldots, a_{k m_{k}}\right)=\Delta\left(a_{11}, \ldots, a_{k m_{k}}\right) \text { and } \\
& \left.t\left(a_{i j}\right)=a_{i j} \text { for } a l l i \leq q\right\} .
\end{aligned}
$$

$\Delta^{t}\left(a_{11}, \ldots, a_{k m_{k}}\right)$ is the result of replacing all accurrencies of $a_{i j}$ in formulas of $\Delta\left(a_{11}, \ldots, a_{k m_{k}}\right)$ by $t\left(a_{i j}\right) ; i=1, \ldots, k$, $j=1, \ldots, m$. Dbviously

$$
G_{0} \supseteq G_{1} \supseteq G_{2} \supseteq \cdots \supseteq G_{k}=\{e\} .
$$


This sequence of groups does not depend on the particular choice of the splitting diagrams $\Delta\left(a_{11}, \ldots, a_{p m p}\right)$. This is shown by induction, using the general fact [1, theorem 1] that any two splitting diagrams are just permutational variants of each other: For every splitting diagram $\Delta^{1}\left(a_{11}, \ldots, a_{1 m_{1}}\right)$ of $\Delta$ there exists a permutation $s$ of $\left\{a_{11}, \ldots, a_{1 m 1}\right\}$ such that $\Delta^{1}\left(a_{11}, \ldots, a_{1 m_{1}}\right)=\Delta^{s}\left(a_{11}, \ldots, a_{1 m_{1}}\right)$.

Our central point here is to investigate relations between the graups $G_{q}, G_{\Delta}(c p)$ and $G_{\Lambda\left(a_{11}, \ldots, a_{p m_{p}}\right)}\left(\psi\left(T_{p+1} v\right)\right)$.

\section{$\stackrel{\operatorname{Lemma}}{=}=\stackrel{1}{=}==$}

$G_{\Delta}(\varphi) \cong\left\{s \in s_{n}: \exists t \in G_{0}\right.$ such that $\left.\Gamma u \Delta\left(a_{11}, \ldots, a_{k m k}\right) \vdash \sigma_{s(i)}=\sigma_{i}^{t}, i=1,2, \ldots, n\right\}$. Proof. Let $\Delta\left(b_{1}, \ldots, b_{n}\right)$ be a splitting diagram of $c(y)$ over $\Delta$. Let $S$ be the set of all permutations of $\left\{b_{1}, \ldots, b_{n}\right\}$. It is clear from the definition that

$$
G=\left\{s \in S: \Delta\left(b_{1}, \ldots, b_{n}\right)=\Delta^{S}\left(b_{1}, \ldots, b_{n}\right)\right\} \cong G_{\Delta}(\varphi(y)) .
$$

Let $\bar{P}$ be the set of all permutations $\bar{t}$ of $\left\{b_{1}, \ldots, b_{n}, a_{11}, \ldots, a_{k m_{k}}\right\}$ such that $\bar{t}$ restricted to $\left\{a_{11}, \ldots, a_{k m_{k}}\right\}$ belongs to $p$. Let

$$
\bar{G}=\left\{\bar{t} \in \bar{P}: \Delta^{\bar{t}}\left(b_{1}, \ldots, b_{n}, a_{11}, \ldots, a_{k m_{k}}\right)=\Delta\left(b_{1}, \ldots, b_{n}, a_{11}, \ldots, a_{k m_{k}}\right)\right\} .
$$

We define a map $f: \bar{G} \rightarrow G$ by

$$
f(\bar{t})\left(b_{i}\right)=\bar{t}\left(b_{i}\right) \text {. }
$$

$f$ is a homomorphism of $\bar{G}$ onto $G$. It is easy to check that $f$ is homomorphic. To prove that $f$ is onto let $s \in G$ be given. Let $श\left(b_{1}, \ldots, b_{n}\right)$ be the minimal model of $\Gamma_{v} \Delta\left(b_{1}, \ldots, b_{n}\right)$, I $\left(b_{1}, \ldots, b_{n}, a_{11}, \ldots, a_{k m_{k}}\right)$ the minimal model of $\Gamma \cup \Delta\left(b_{1}, \ldots, b_{n}, a_{11}, \ldots a_{k m}{ }_{k}\right.$ Consider the following application of the amalgamation property: 


$$
\begin{aligned}
& \mathscr{I s}\left(b_{1}, \ldots, b_{n}\right) \hookrightarrow \mathscr{U}\left(b_{1}, \ldots, b_{n}, a_{11}, \ldots, a_{k m_{k}}\right) \\
& \left.\mathcal{U ( b}, \ldots, b_{\Pi}\right) \rightarrow \text { c }
\end{aligned}
$$

(5) can be chosen as an extension of $\mathfrak{U}\left(b_{1}, \ldots, b_{n}, a_{11}, \ldots, a_{k m_{k}}\right)$. Since $\left\{b_{1}, \ldots, b_{n}\right\}$ are all of the possible solutions of $\varphi(y)$, in all extensions, and $\bar{t}_{s}$ is monomorphic, $\bar{t}_{s}$ must permute $\left\{b_{1}, \ldots, b_{n}\right\}$. By the same reasoning $\bar{t}_{s}$ permutes each $\left\{a_{q 1}, \ldots, a_{q m_{q}}\right\}$. Thus, is can be chosen as $2\left(a_{1}, \ldots, b_{n}, a_{11}, \ldots, a_{k m_{k}}\right)$ and $\bar{t}_{s} \in \bar{G}$ is onto.

\{we are using the same letters to denote the permutation of the individual constants and the corresponding automorphisms of the models; which meaning is chosen is clear from the context\}. Define $g: G_{0} \rightarrow G$ by $g(t)=f(\bar{t})$, where $\bar{t}$ is the extension of $t$ to $\left\{b_{1}, \ldots, b_{n}\right\}$ defined by $\bar{t}\left(b_{i}\right)=\sigma_{i}^{t}, i=1, \ldots, n$. The map $g$ is obviously a homomorphism onto $G$. Hence $s \in G$ iff there exists $t \in G_{0}$ such that $g(t)=s$. Observe $g(t)=s$ iff $g(t)\left(b_{i}\right)=s\left(b_{i}\right)$ $\operatorname{for}^{\circ} i=1, \ldots, n$, iff $\Gamma \cup \Delta\left(a_{11}, \ldots, a_{k m_{k}}\right) \vdash s\left(\sigma_{i}\right)=\sigma_{i}^{t}$ for $i=1, \ldots, n$. It follows that

$G_{\Delta}(\varphi) \cong G=\left\{s \in S: \exists t \in G\right.$ with $\left.\Gamma u \Delta\left(a_{11}, \ldots, a_{k m_{k}}\right)+s\left(\sigma_{i}\right)=\sigma_{i}{ }^{t}\right\}$ $\cong\left\{s \in S_{n}: \exists t \in G_{0}\right.$ with $\left.\Gamma v \Delta\left(a_{11}, \ldots, a_{k m_{k}}\right) \sigma_{s(i)}=\sigma_{i}{ }^{t}\right\}$.

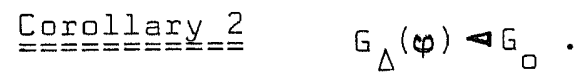

Lemma $==3 \quad G_{q+1} \triangleleft G_{q}, q=0,1, \ldots, k-1$.

Proof. Let $s \in G_{q}, t \in G_{q+1}$. We show $s^{-1} t s \in G_{q}$.

Clearly $\Delta^{s^{-1} t s}\left(a_{11}, \ldots, a_{k m_{k}}\right)=\Delta\left(a_{11}, \ldots, a_{k m_{k}}\right)$. Furthermore, for all $q$ and $j$ there exists $p$ such that

$$
s^{-1} t s\left(a_{q+1 j}\right)=s^{-1} t\left(a_{q+1 p}\right)=s^{-1}\left(a_{q+1 p}\right)=a_{q+1 j} \text {. }
$$

$\stackrel{\text { Lemma }}{=}=\stackrel{4}{=}=\stackrel{4}{=}$

$$
G_{q} / G_{q+1} \cong G_{\Delta\left(a_{11}, \ldots, a_{q m_{q}}\right)}\left(\psi\left(\tau_{q+1}, v\right)\right), q=0,1, \ldots, k-1 .
$$


Proof. Let $S$ be the set of all permutations of

Then

$$
\left\{a_{q+1}, \ldots, a_{q+1, m_{q+1}}\right\} \text {. }
$$

$G_{\Lambda\left(a_{11}, \ldots, a_{q m_{q}}\right)}\left(\psi\left(T_{q+1}, v\right)\right)=\left\{s \in 5: \Delta s\left(a_{11}, \ldots, a_{q+1 m_{q+1}}\right)=\Delta\left(a_{11}, \ldots, a_{q+1 m_{q+1}}\right)\right\}_{.}$

We construct an isomorphism $f$ of $G_{\Delta\left(a_{11}, \ldots, a_{q m_{q}}\right)}\left(\psi\left(\tau_{q+1}, v\right)\right)$ onto $G_{q} / G_{q+1}$ as follows: Let $s \in G_{\Delta\left(a_{11}, \ldots, a_{q m_{q}}\right)}\left(\psi\left(\tau_{q+1}, v\right)\right)$, and let $\mathscr{U}\left(a_{1}, \ldots, a_{k m_{k}}\right)$ be the minimal model of $\Gamma u\left(a_{11}, \ldots, a_{k m k}\right)$. Let $t$ be any extension of $s$ to an automorphism of $\mathscr{U}\left(a_{11}, \ldots, a_{k m_{k}}\right)$ leaving $\mathscr{U}\left(a_{11}, \ldots, a_{q_{m}}\right)$ pointwise fixed. Then we define $f(s)=t E_{q+1}$.

(a) The existence of $t$ is proved in the same way as that of $\bar{t}_{s}$ in the proof of Lemma 1 .

(b) $f$ is well-defined, i.e. it does not depend an the choice of $t$ : Let $t_{1}, t_{2}$ be extensions of $s$. Consider $u=t_{2}^{-1} t$. We need to show $u(x)=x$ for all $x \in(i)\left(a_{11}, \ldots, a_{q m_{q}}\right)$. But $t_{2}^{-1} t(x)=t_{2}^{-1} s(x)=s^{-1} s(x)=x$, since $s(x) \in \mathbb{M}\left(a_{11}, \ldots, a_{q m_{q}}\right)$.

(c) $f$ is one-to-one: Assume $f\left(s_{1}\right)=f\left(s_{2}\right)$, i.e. $t_{1} G_{q+1}=t_{2} G_{q+1}$. Then $t_{1} \in t_{2} G_{q+1}$ and $t_{1}=t_{2} y$ for some $y \in G_{q+1}$. For an arbitrary element $x \in \mathbb{U}\left(a_{11}, \ldots, a_{q+1 m_{q+1}}\right)$ we have then $s_{1}(x)=t_{1}(x)=\left(t_{2} y\right)(x)=t_{2}(y(x))=t_{2}(x)=s_{2}(x)$. Hence $s_{1}=s_{2}$.

(d) $f$ is anto: Let $t G_{q+1} \in G_{q} / G_{q+1}$, $t \in G_{q}$ be given. Let $t^{1}$ be the restriction of $t$ to $^{q}\left\{a_{11}, \ldots, a_{q+1 m_{q+1}}\right\}$. Then $\Delta^{t^{1}}\left(a_{11}, \ldots . a_{q+1} m_{q+1}\right)=\Delta^{t}\left(a_{11}, \ldots, a_{q+1 m_{q+1}}\right)=\Delta\left(a_{11}, \ldots, a_{q+1} m_{q+1}\right)$.

In addition, $t^{1}\left(a_{i j}\right)=a_{i j}$ for all $i \leq q$, since $t \in G_{q}$. Therefore $\quad t^{1} \in G\left(\mathfrak{H}\left(a_{11}, \ldots, a_{q+1 m_{q+1}}\right) / \mathfrak{M}\left(a_{11}, \ldots, a_{q m_{q}}\right)\right) \cong$

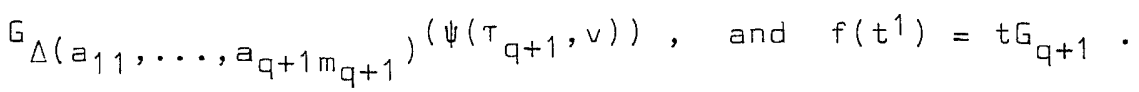

(e) $f$ is a homomorphism: Because $G_{q+1} \triangleleft G_{q}$ we may compute $f\left(s_{1}\right) \cdot f\left(s_{2}\right)=t_{1} G_{q+1} t_{2} G_{q+1}=t_{1}\left(t_{2} G_{q+1} t_{2}^{-1}\right) t_{2} G_{q+1}=t_{1} t_{2} G_{q+1}=f\left(s_{1} \cdot s_{2}\right)$. 
The last equality follows from the fact that if $t_{1}, t_{2}$ are extensions of $s_{1}, s_{2}$ then $t_{1} \cdot t_{2}$ is an extension of $s_{1} \cdot s_{2}$. Namely, $\quad t_{1} t_{2}\left(a_{q+1 j}\right)=t_{1} s_{2}\left(a_{q+1 j}\right)=s_{1} s_{2}\left(a_{q+1 j}\right)$ because $s_{2}\left(a_{q+1 j}\right)$ is of the form $a_{q+1 p} \square$.

4. Complexity

There are two tasks still before us. One, to use the above results to obtain our complexity theorem, the other to discuss effective methods for obtaining the group of a problem. Lemma 4 implies (using the notational conventions of section 3):

$$
\begin{aligned}
& \left|G_{q}\right|=\left|G_{q+1}\right| \cdot\left|G_{\Delta\left(a_{11}, \ldots, a_{q m_{q}}\right)}\left(\psi\left(T_{q+1}, v\right)\right)\right|, q=0,1, \ldots, k-1, \\
& \text { since }\left|G_{k}\right|=|\{e\}|=1 \text {, we have therefore } \\
& \left|G_{\square}\right|=\prod_{q=0}^{k-1} \mid G_{\Delta}\left(a_{11}, \ldots, a_{q m_{q}}\right) \\
& \left|G_{\Delta}(c p)\right| \leq \prod_{q=0}^{k-1}\left|G_{\Delta\left(a_{11}, \ldots, a_{q m_{q}}\right)}\left(\psi\left(\tau_{q+1}, v\right)\right)\right| .
\end{aligned}
$$

We shall use this inequality to obtain a lower bound for the complexity of $\varphi(y)$.

Assume, then, that $\varphi(y)$ can be solved relative to $\psi(u, v)$ in all madels of $\Gamma \Delta$ by a program $\pi$. The complexity of $\pi$ alang a path $w$ is the number of times an auxillary problem $\psi$ needs to be solved along $w$ until all solutions of $\varphi(y)$ are obtained. \{Thus we are looking for worst-case behaviour\}. The complexity of $\pi$ is the minimal complexity of $\pi$ along paths. The complexity of $\varphi(y)$ is the minimal complexity of solution algorithms $\pi$ relative to auxillary problems $\psi$.

\section{Theorem}

The complexity of $\varphi(y)$ has a lower bound $\log _{b}\left|G_{\Delta}(\varphi)\right|$, where $b=\max \left\{\left|G_{\Delta(a)}(\psi(a, v))\right|: \operatorname{\Gamma u} \Delta(a)\right.$ consistent, $\left.\Delta(a) \supseteq \Delta\right\}>1$. 
Proof. Let $\pi$ be an arbitrary solution algorithm for $\varphi(y)$ relative to $\psi(u, v)$ and $w$ a path through $\pi$ constistent with rus. Let $k$ be the number of times $\psi$ needs to be solved along $w$. We are looking for a lower bound to $k$ independent of $\pi$ and $w$. Define $\alpha_{q}=1 / \log \left|G_{\Delta\left(a_{11}, \ldots, a_{q m_{q}}\right)}\left(\psi\left(\tau_{q+1}, v\right)\right)\right|, q=0,1, \ldots, k-1$.
Then:

$$
\begin{aligned}
& k=\sum_{q=0}^{k-1} \alpha_{q} \cdot \log \left|G_{\Delta\left(a_{1}, \ldots, a_{q m_{q}}\right)}\left(\psi\left(\tau_{q+1}, v\right)\right)\right| \\
& \left.\geq \min _{q=0, \ldots, k-1}\left(\alpha_{q}\right) \cdot \sum_{q=0}^{k-1} \log \mid G_{\Delta a_{11}}, \ldots, a_{q m_{q}}\right)\left(\psi\left(\tau_{q+1}, v\right)\right) \mid
\end{aligned}
$$

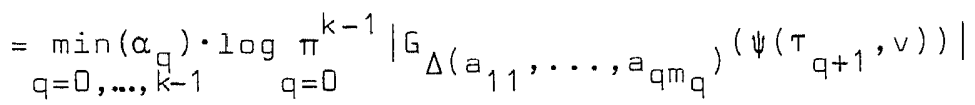

$$
\begin{aligned}
& =\underset{q=0, \ldots, k-1}{\min \left(\alpha_{q}\right) \cdot \log \left|G_{0}\right| \geq \min _{q=0, \ldots, k-1}\left(\alpha_{q}\right) \cdot \log \left|G_{\triangle}(s)\right|} \\
& =\log \left|G_{\Delta}(\varphi)\right| / \log \max _{q=0, \ldots, k-1}\left|G_{\Delta\left(a_{11}, \ldots, a_{q m_{q}}\right)}\left(\psi\left(\tau_{q+1}, v\right)\right)\right| \\
& \geq \log \left|G_{\Delta}(\varphi)\right| / \log b=\log _{b}\left|G_{\Delta}(\varphi)\right| \text {. }
\end{aligned}
$$

The theorem obviously generalizes to more than one auxillary problem and to problems of the form $\varphi\left(y_{1}, \ldots, y_{k}\right)$.

How should we determine $G_{\Delta}(\varphi)$ ?

It is reasonable to assume that we are given a solution alqarithm $\pi$ of $\varphi(y)$ relative to $\psi(u, v)$ which terminates in all models of $\Gamma v \Delta$. Let us assume, in addition, that the universal theory of Iu is decidable, i.e. that for every quantifier-free formula $x\left(x_{1}, \ldots, x_{m}\right)$ of $L$ we can decide whether

$$
\Gamma v \Delta \mid \forall x_{1}, \ldots, x_{m} x\left(x_{1}, \ldots, x_{m}\right) \text {. }
$$

It fallows, that for additional constants $a_{1}, \ldots, a_{m}$ we can decide whether

$\Gamma \cup \Delta \quad\left\{x\left(a_{1}, \ldots, a_{m}\right)\right\}$

is consistent. This fact can be used to effectively find a consistent finite path through $\pi$. 
The initial part of the path, up to the first time a problem $\psi$ has to be solved is obviously determined by $\Delta$ alone. Let $\psi(T, v)$ be the first problem, $T_{1}$ a constant term of $L$. We need to know the degree of $\Delta$ for $\psi(\tau, v)$. This is the maximal $m$, such that

$$
\Gamma u \Delta\left\{\psi\left(\tau_{1}, a_{1 j}\right)\right\}_{j=1}^{m_{1}} \vee\left\{a_{1 j} \neq a_{1 k}\right\}_{j \neq k}
$$

is consistent. By assumption, this number $m$, can be effectively found. Progressing further along the path, we need to decide relations $R\left(\mu_{1}, \ldots, \mu_{k}\right)$, where the $\mu_{j}$ are constant terms of $L\left(a_{11}, \ldots, a_{1 m_{1}}\right)$. Using the assumption again, we are able to augment the previously extended set consistently in such a fashion that the resulting set of formulas determines the path up to the next time $\psi$ needs tobe solved, say for $\psi\left(\tau_{2}, v\right)$, $\tau_{2}$ a constant term of $L\left(a_{11}, \ldots, a_{1 m_{1}}\right)$. Again, we determine $m_{2}$, etc. This process if repeated, finitely often by assumption on the termination of $\pi$, until we reach the end of the path. At that point we have collected, by conjunction, a finite quantifier-free formula $\delta\left(a_{11}, \ldots, a_{k m_{k}}\right)$ and have determined constant terms $\sigma_{1}, \ldots, \sigma_{n}$ of $L\left(a_{11}, \ldots, a_{k m_{k}}\right)$ such that

$$
\begin{aligned}
& \Gamma \cup \Delta \cup\left\{\delta\left(a_{11}, \ldots, a_{k m_{k}}\right)\right\} \quad \text { is consistent; } \\
& \Gamma \cup \Delta \cup\left\{\delta\left(a_{11}, \ldots, a_{k m_{k}}\right)\right\} \mapsto \varphi\left(\sigma_{i}\right), i=1, \ldots, n ; \\
& \Gamma \cup \Delta \cup\left\{\delta\left(a_{11}, \ldots, a_{k m_{k}}\right)\right\} \vdash \sigma_{i} \neq \sigma_{j}, i \neq j .
\end{aligned}
$$

We use Lemma 1 to compute $G_{\Delta}(\varphi)$. For this we consider

$$
G_{0}=\left\{t \in P: \Delta t\left(a_{11}, \ldots, a_{k m_{k}}\right)=\Delta\left(a_{11}, \ldots, a_{k m_{k}}\right)\right\} .
$$

Of $\Delta\left(a_{11}, \ldots, a_{k m_{k}}\right)$ which enters the definition of $G_{0}$ we only know the finite subset whose conjunction is $\delta\left(a_{11}, \ldots, a_{k m_{k}}\right)$. This formula, however determines $\Delta\left(a_{11}, \ldots a_{k m_{k}}\right.$ ) completely (up to a permutation of the $\left.a_{i j}\right)$ because it forces the $\Delta\left(a_{11}, \ldots, a_{q m_{q}}\right)$ to be splitting diagrams. Thus $G_{0}$ is completely determined by

$$
\Gamma \cup \Delta \cup\left\{\delta\left(a_{11}, \ldots, a_{k m_{k}}\right)\right\} \text {. }
$$

Assume that $G_{0}$ is known. 
To obtain $G_{\Delta}(\varphi)$ we extend the last set of formulas cansitently until, for all $i, j \leq n$ and all $t \in G_{0}$ we have decided the equation

$$
\sigma_{i}=\sigma_{j}^{t}
$$

Altogether, we have shown, that under the stated assumptions the group $G_{\Delta}(\varphi)$ can be effectively determined. We are not claimed that our algorithm is always the best one, or even feasible. The assumptions, however, seem reasonable: That a relative solution algorithm be known, that the theory have the basic decidability property stated and that the groups of the auxillary problems $\psi(\tau, v)$ be known for "algebraic" elements $\tau$.

5. Remarks

(1) The theorem gives an exact formulation to the following abservation: A problem grows harder to solve if less properties can be found which distinguish its individual solutions. - This suggests, that in the search for large lower complexity bounds it is reasonable to formulate those special cases where the set of solutions admits as many symmetries as possible.

(2) We are possibly losing some of the complexity information by computing $\log _{b}\left|G_{\Delta}(\varphi)\right|$ instead of $\log _{b}\left|G_{a}\right|$, since $G_{\Delta}(\varphi) \Delta G_{0}$. The index of $G_{\Delta}(\varphi)$ in $G_{0}$ is a measure for the directmess of the relative solution algorithm. If this index is one, then each admissible permutation of the $a_{i j}$ is determined by an admissible permutation of the $b_{i}$. This is true, like in the classical case of Galois theory, if the $a_{i j}$ can be expressed as terms in the $b_{i}$.

(3) It is easy to produce examples which show that the full 'fundamental theorem of Galois theory' does not generalize without additional assumptions: Not to every (normal) subgroup of the group of a problem does there exist a corresponding submodel. 
[1] Engeler, E.: 1975, 'Dn the Solvability of Algorithmic Problems', in H.E. Rose and J.C. Shepherdson (eds.), Loqic Colloquium '73, North-Holland Publ. Co., 1975, pp. $231-251$.

[2] Jónsson, B.: 1962, 'Algebraic Extensions of Relational Systems', Mathematica Scandinavica 11, pp. 179-205.

Address of the author:

Prof. Dr. E. Engeler, E.T.H.

Clausiusstr. 55

8006 Zürich, Switzerland 